\title{
Approximate controllability of a class of coupled degenerate systems
}

\author{
Yingjie Zhu', Runmei Du ${ }^{2 *}$ and Lianzhang $\mathrm{BaO}^{3}$
}

\section{"Correspondence:}

durm_dudu@163.com

${ }^{2}$ School of Basic Science,

Changchun University of

Technology, Changchun, 130012,

P.R. China

Full list of author information is

available at the end of the article

\begin{abstract}
This paper concerns the approximate controllability of a class systems governed by coupled degenerate equations. The equations may be weakly degenerate and strongly degenerate on the boundary. It is shown that the systems are approximately controllable by constructing the controls via the conjugate problems.
\end{abstract}

MSC: 93B05; 93C20; 35K65

Keywords: approximate controllability; degenerate system; coupled equations

\section{Introduction}

In this paper, we investigate the approximate controllability of the coupled degenerate parabolic equations

$$
\begin{array}{ll}
\frac{\partial y}{\partial t}-\operatorname{div}\left(a_{1}(x, t) \nabla y\right)+c_{1}(x, t) y=h(x, t) \chi_{\omega_{1}}, & (x, t) \in Q_{T}, \\
\frac{\partial u}{\partial t}-\operatorname{div}\left(a_{2}(x, t) \nabla u\right)+c_{2}(x, t) u=y(x, t) \chi_{\omega_{2}}, & (x, t) \in Q_{T},
\end{array}
$$

where $Q_{T}=\Omega \times(0, T), \Omega$ is a bounded domain in $\mathbb{R}^{n}, T>0, h \in L^{2}\left(Q_{T}\right)$ is the control function, $\chi$ is the characteristic function, $\omega_{1}$ and $\omega_{2}$ are open subsets of $\Omega$ satisfying $\omega_{1} \cap \omega_{2} \neq \emptyset, a_{j} \in C\left(\bar{Q}_{T}\right)$ is positive in $\Omega \times(0, T)$ with $\frac{1}{a_{j}} \frac{\partial a_{j}}{\partial t} \in L^{\infty}\left(Q_{T}\right)$ and $c_{j} \in L^{\infty}\left(Q_{T}\right)$ for $j=1,2$.

Equations (1.1) and (1.2) can be used to describe some physical models. For instance, in [1] we can find a motivating example of a Crocco-type equation coming from the study of the velocity field of laminar flow on a flat plate. It is noted that (1.1) and (1.2) may be degenerate at some points on $\partial \Omega \times(0, T)$. According to [2], we can prescribe the following boundary and initial values:

$$
\begin{aligned}
& y(x, t)=0, \quad(x, t) \in \Sigma_{1}, \\
& u(x, t)=0, \quad(x, t) \in \Sigma_{2}, \\
& y(x, 0)=y_{0}(x), \quad x \in \Omega, \\
& u(x, 0)=u_{0}(x), \quad x \in \Omega,
\end{aligned}
$$

(c) 2016 Zhu et al. This article is distributed under the terms of the Creative Commons Attribution 4.0 International License (http://creativecommons.org/licenses/by/4.0/), which permits unrestricted use, distribution, and reproduction in any medium, provided you give appropriate credit to the original author(s) and the source, provide a link to the Creative Commons license, and indicate if changes were made. 
where $y_{0}, u_{0} \in L^{2}(\Omega)$ and

$$
\begin{aligned}
\Sigma_{j}= & \{(x, t) \in \partial \Omega \times(0, T): \text { there exists } 0<\delta<\min \{t, T-t\} \text { such that } \\
& \left.\int_{t-\delta}^{t+\delta} \int_{\{y \in \Omega:|y-x|<\delta\}} \frac{1}{a_{j}(y, s)} d y d s<+\infty\right\}, \quad j=1,2 .
\end{aligned}
$$

Note that $\Sigma_{j}$ denotes the nondegenerate and weak degenerate part of the lateral boundary, which does not include the strong degenerate part. For example, if $n=1, \Omega=(0,1)$, $a_{1}(x, t)=a_{2}(x, t)=x^{\alpha}$, then if $\alpha=0$, the boundary $x=0$ is nondegenerate part; if $0<\alpha<1$, the boundary $x=0$ is weak degenerate part; if $\alpha \geq 1$, the boundary $x=0$ is strong degenerate part. When $\Sigma_{j}=\emptyset$, the equations are in strong degeneracy at each point of the lateral boundary.

Controllability theory has been widely investigated for the systems governed by nondegenerate parabolic equations over the last 40 years and there have been a great number of results (see for instance [3-5] and the references therein for a detailed account). However, the study of the systems governed by degenerate parabolic equations just began several years ago and there are some results (see [6-16] and the references therein). Different from nondegenerate parabolic equations, the null controllability and the approximate controllability for the systems governed by degenerate parabolic equations may be inconsistent. Indeed, if $n=1, \Omega=(0,1)$, and

$$
a_{1}(x, t)=x^{\alpha}, \quad(x, t) \in Q_{T}(\alpha>0),
$$

it is shown that the system (1.1), (1.3), (1.5) is null controllable if $0<\alpha<2[6,9,10,14]$, while not if $\alpha \geq 2$ [11], while it is approximately controllable for $\alpha>0$ [15, 16]. More generally, the authors $[15,16]$ proved the approximate controllability of the system (1.1), (1.3), (1.5) governed by one single equation in the multi-dimensional case. Besides, $[12,13]$ are concerned with the null controllability of the degenerate coupled equations. Particularly, the authors studied the null controllability of the system (1.1)-(1.6) for the special case that

$$
n=1, \quad \Omega=(0,1) \quad \text { and } \quad a_{1}(x, t)=a_{2}(x, t)=x^{\alpha} \quad(\alpha>0)
$$

and showed that the system is null controllable if $0<\alpha<2$ in [12].

In the present paper, we prove the approximate controllability of the system (1.1)-(1.6). That is to say, for any admissible error value $\varepsilon>0$ and the desired datum $\left(y_{d}, u_{d}\right) \in L^{2}(\Omega) \times$ $L^{2}(\Omega)$, there exists a control function $h$ such that the solution $(y, u)$ to the problem (1.1)(1.6) approximately approaches $\left(y_{d}, u_{d}\right)$ at time $T$, i.e.

$$
\left\|y(\cdot, T)-y_{d}(\cdot)\right\|_{L^{2}(\Omega)} \leq \varepsilon, \quad\left\|u(\cdot, T)-u_{d}(\cdot)\right\|_{L^{2}(\Omega)} \leq \varepsilon
$$

\section{Well-posedness and approximate controllability}

The solutions to the problem (1.1)-(1.6) are defined as follows. 
Definition 2.1 A pair of functions $(y, u)$ is called a weak solution to the problem (1.1)-(1.6), if $y \in C\left([0, T] ; L^{2}(\Omega)\right) \cap \mathscr{B}_{1}, u \in C\left([0, T] ; L^{2}(\Omega)\right) \cap \mathscr{B}_{2}$ satisfy

$$
\begin{aligned}
& \iint_{Q_{T}}\left(-y \frac{\partial \varphi}{\partial t}+a_{1} \nabla y \cdot \nabla \varphi+c_{1} y \varphi\right) d x d t-\int_{\Omega} y_{0}(x) \varphi(x, 0) d x=\iint_{Q_{T}} h \chi_{\omega_{1}} \varphi d x d t \\
& \iint_{Q_{T}}\left(-u \frac{\partial \psi}{\partial t}+a_{2} \nabla u \cdot \nabla \psi+c_{2} u \psi\right) d x d t-\int_{\Omega} u_{0}(x) \psi(x, 0) d x=\iint_{Q_{T}} y \chi_{\omega_{2}} \psi d x d t
\end{aligned}
$$

for any $\varphi \in L^{\infty}\left((0, T) ; L^{2}(\Omega)\right) \cap \mathscr{B}_{1}, \psi \in L^{\infty}\left((0, T) ; L^{2}(\Omega)\right) \cap \mathscr{B}_{2}$ with $\frac{\partial \varphi}{\partial t}, \frac{\partial \psi}{\partial t} \in L^{2}\left(Q_{T}\right)$ and $\left.\varphi(\cdot, T)\right|_{\Omega}=\left.\psi(\cdot, T)\right|_{\Omega}=0$. Here, $\mathscr{B}_{j}$ is the closure of $C_{0}^{\infty}\left(Q_{T}\right)$ with respect to the norm

$$
\|w\|_{\mathscr{B}_{j}}=\left(\iint_{Q_{T}} a_{j}(x, t)\left((w(x, t))^{2}+|\nabla w(x, t)|^{2}\right) d x d t\right)^{1 / 2}, \quad w \in \mathscr{B}_{j}
$$

for $j=1,2$.

Similar to the single equation case (Theorem 2.1 in [15]), one can prove the following well-posedness.

Theorem 2.1 Assume $a_{j} \in C\left(\bar{Q}_{T}\right)$ is positive in $\Omega \times(0, T)$ with $\frac{1}{a_{j}} \frac{\partial a_{j}}{\partial t} \in L^{\infty}\left(Q_{T}\right)$ and $c_{j} \in$ $L^{\infty}\left(Q_{T}\right)$ for $j=1,2$. Then for any $h \in L^{2}\left(Q_{T}\right)$ and $y_{0}, u_{0} \in L^{2}(\Omega)$, the problem (1.1)-(1.6) admits uniquely a weak solution $(y, u)$. Furthermore, the solution $(y, u)$ satisfies

$$
\begin{aligned}
& \|y\|_{L^{\infty}\left((0, T) ; L^{2}(\Omega)\right)}+\|u\|_{L^{\infty}\left((0, T) ; L^{2}(\Omega)\right)}+\left\|a_{1}|\nabla y|^{2}\right\|_{L^{1}\left(Q_{T}\right)}+\left\|a_{2}|\nabla u|^{2}\right\|_{L^{1}\left(Q_{T}\right)} \\
& \quad \leq C\left(\|h\|_{L^{2}\left(Q_{T}\right)}+\left\|y_{0}\right\|_{L^{2}(\Omega)}+\left\|u_{0}\right\|_{L^{2}(\Omega)}\right)
\end{aligned}
$$

where $C>0$ depends only on $\Omega, T,\left\|c_{1}\right\|_{L^{\infty}\left(Q_{T}\right)}$, and $\left\|c_{2}\right\|_{L^{\infty}\left(Q_{T}\right)}$.

Remark 2.1 If $u \in \mathscr{B}_{j}$, then $\left.u\right|_{\Sigma_{j}}=0$ in the trace sense, while there is no trace on $(\partial \Omega \times$ $(0, T)) \backslash \Sigma_{j}$ in general.

The study of the approximate controllability of the system (1.1)-(1.6) is related to its conjugate system

$$
\begin{aligned}
& -\frac{\partial z}{\partial t}-\operatorname{div}\left(a_{1}(x, t) \nabla z\right)+c_{1}(x, t) z=v \chi_{\omega_{2}}, \quad(x, t) \in Q_{T}, \\
& -\frac{\partial v}{\partial t}-\operatorname{div}\left(a_{2}(x, t) \nabla v\right)+c_{2}(x, t) v=0, \quad(x, t) \in Q_{T}, \\
& z(x, t)=0, \quad(x, t) \in \Sigma_{1}, \\
& v(x, t)=0, \quad(x, t) \in \Sigma_{2}, \\
& z(x, T)=z_{0}(x), \quad x \in \Omega, \\
& v(x, T)=v_{0}(x), \quad x \in \Omega .
\end{aligned}
$$

Define the mapping

$$
\mathscr{L}: \mathscr{H} \rightarrow L^{2}\left(\omega_{1} \times(0, T)\right), \quad\left(z_{0}, v_{0}\right) \longmapsto z \chi_{\omega_{1}},
$$


where $\mathscr{H}=L^{2}(\Omega) \times L^{2}(\Omega)$ with the norm

$$
\left\|\left(w_{1}, w_{2}\right)\right\|_{\mathscr{H}}=\left(\left\|w_{1}\right\|_{L^{2}(\Omega)}^{2}+\left\|w_{2}\right\|_{L^{2}(\Omega)}^{2}\right)^{1 / 2}, \quad\left(w_{1}, w_{2}\right) \in \mathscr{H} .
$$

Proposition 2.1 The conjugate problem (2.1)-(2.6) possesses the property of unique continuation. That is to say, if $z=0$ a.e. in $\omega_{1} \times(0, T)$, then $z=v=0$ a.e. in $Q_{T}$.

Proof From (2.1) and $z=0$ a.e. in $\omega_{1} \times(0, T)$, one gets $v=0$ a.e. in $\left(\omega_{1} \cap \omega_{2}\right) \times(0, T)$. For sufficiently small $\delta>0$, denote

$$
\Omega_{\delta}=\{x \in \Omega: \operatorname{dist}(x, \partial \Omega)>\delta\} .
$$

Since (2.2) is nondegenerate in $\Omega_{\delta} \times(0, T)$, one gets from the classical unique continuation [17] $v=0$ a.e. in $\Omega_{\delta} \times(0, T)$. It follows from the arbitrariness of $\delta$ that $v=0$ a.e. in $Q_{T}$, which also shows that $z$ satisfies the homogeneous equation. Then the same discussion as for $v$ leads to $z=0$ a.e. in $Q_{T}$.

Define the functional

$$
\begin{aligned}
J\left(\left(z_{0}, v_{0}\right)\right)= & \frac{1}{2} \int_{0}^{T} \int_{\omega_{1}}\left|\mathscr{L}\left(\left(z_{0}, v_{0}\right)\right)(x, t)\right|^{2} d x d t+\varepsilon\left\|\left(z_{0}, v_{0}\right)\right\|_{\mathscr{H}} \\
& -\left\langle\left(y_{d}, u_{d}\right),\left(z_{0}, v_{0}\right)\right\rangle_{\mathscr{H}}, \quad\left(z_{0}, v_{0}\right) \in \mathscr{H},
\end{aligned}
$$

where $\langle(\cdot, \cdot),(\cdot, \cdot)\rangle_{\mathscr{H}}$ is the inner product in $\mathscr{H}$.

Proposition 2.2 J(.) is strictly convex and satisfies

$$
\lim _{\left\|\left(z_{0}, v_{0}\right)\right\| \mathscr{H} \rightarrow+\infty} \inf \frac{J\left(\left(z_{0}, v_{0}\right)\right)}{\left\|\left(z_{0}, v_{0}\right)\right\|_{\mathscr{H}}} \geq \varepsilon .
$$

Furthermore, $J(\cdot)$ reaches its minimum at a unique point $\left(\hat{z}_{0}, \hat{v}_{0}\right)$ in $\mathscr{H}$ and

$$
\left(\hat{z}_{0}, \hat{v}_{0}\right)=0 \quad \text { a.e. in } \Omega \quad \Longleftrightarrow\left\|\left(y_{d}, u_{d}\right)\right\|_{\mathscr{H}} \leq \varepsilon \text {. }
$$

Proof Note that $\mathscr{L}$ is a linear operator, one can easily prove that $J(\cdot)$ is strictly convex and continuous. Now we prove (2.7) by contradiction. Otherwise, there exists a sequence $\left\{\left(z_{0}^{(k)}, v_{0}^{(k)}\right)\right\}_{k=1}^{\infty} \subset \mathscr{H}$ satisfying

$$
\lim _{k \rightarrow \infty}\left\|\left(z_{0}^{(k)}, v_{0}^{(k)}\right)\right\|_{\mathscr{H}}=+\infty, \quad \lim _{k \rightarrow \infty} \frac{J\left(\left(z_{0}^{(k)}, v_{0}^{(k)}\right)\right)}{\left\|\left(z_{0}^{(k)}, v_{0}^{(k)}\right)\right\|_{\mathscr{H}}}<\varepsilon .
$$

Define

$$
\left(\tilde{z}_{0}^{(k)}, \tilde{v}_{0}^{(k)}\right)=\frac{\left(z_{0}^{(k)}, v_{0}^{(k)}\right)}{\left\|\left(z_{0}^{(k)}, v_{0}^{(k)}\right)\right\|_{\mathscr{H}}}, \quad k=1,2, \ldots
$$

There exists a subsequence of $\left\{\left(\tilde{z}_{0}^{(k)}, \tilde{v}_{0}^{(k)}\right)\right\}_{k=1}^{\infty}$, denoted like the sequence for convenience, which weakly converges in $\mathscr{H}$ to a function $\left(\tilde{z}_{0}, \tilde{v}_{0}\right) \in \mathscr{H}$ with $\left\|\left(\tilde{z}_{0}, \tilde{v}_{0}\right)\right\|_{\mathscr{H}} \leq 1$. Denote by $(\tilde{z}, \tilde{v})$ and $\left(\tilde{z}^{(k)}, \tilde{v}^{(k)}\right)$ the weak solutions of the conjugate problem (2.1)-(2.6) with 
$\left(z_{0}, v_{0}\right)=\left(\tilde{z}_{0}, \tilde{v}_{0}\right)$ and $\left(z_{0}, v_{0}\right)=\left(\tilde{z}_{0}^{(k)}, \tilde{v}_{0}^{(k)}\right)$, respectively. Then it follows from Theorem 2.1 that $\left(\tilde{z}^{(k)}, \tilde{v}^{(k)}\right)$ converges weakly in $\mathscr{H}$ to $(\tilde{z}, \tilde{v})$. Additionally, (2.9) yields

$$
\lim _{k \rightarrow \infty} \int_{0}^{T} \int_{\omega_{1}}\left|\mathscr{L}\left(\left(\tilde{z}_{0}^{(k)}, \tilde{v}_{0}^{(k)}\right)\right)(x, t)\right|^{2} d x d t=0
$$

Hence

$$
\int_{0}^{T} \int_{\omega_{1}}\left|\mathscr{L}\left(\left(\tilde{z}_{0}, \tilde{v}_{0}\right)\right)(x, t)\right|^{2} d x d t \leq \liminf _{k \rightarrow \infty} \int_{0}^{T} \int_{\omega_{1}}\left|\mathscr{L}\left(\left(\tilde{z}_{0}^{(k)}, \tilde{v}_{0}^{(k)}\right)\right)(x, t)\right|^{2} d x d t=0
$$

which, together with Proposition 2.1 , leads to $(\tilde{z}, \tilde{v})=(0,0)$ in $Q_{T}$ and thus $\left(\tilde{z}_{0}, \tilde{v}_{0}\right)=(0,0)$ in $\Omega$. Thus

$$
\lim _{k \rightarrow \infty} \frac{J\left(\left(z_{0}^{(k)}, v_{0}^{(k)}\right)\right)}{\left\|\left(z_{0}^{(k)}, v_{0}^{(k)}\right)\right\|_{\mathscr{H}}} \geq \varepsilon-\lim _{k \rightarrow \infty} \frac{\left\langle\left(y_{d}, u_{d}\right),\left(z_{0}^{(k)}, v_{0}^{(k)}\right)\right\rangle_{\mathscr{H}}}{\left\|\left(z_{0}^{(k)}, v_{0}^{(k)}\right)\right\|_{\mathscr{H}}}=\varepsilon
$$

which contradicts (2.9) and completes the proof of (2.7).

From (2.7), the strict convexity and the continuity of $J(\cdot), J(\cdot)$ must achieve its minimum at a unique point in $\mathscr{H}$.

Finally, we prove (2.8). On the one hand, if $\left\|\left(y_{d}, u_{d}\right)\right\|_{\mathscr{H}} \leq \varepsilon$, it follows from the Hölder inequality that

$$
J\left(\left(z_{0}, v_{0}\right)\right) \geq 0, \quad\left(z_{0}, v_{0}\right) \in \mathscr{H}
$$

and thus $\left(\hat{z}_{0}, \hat{v}_{0}\right)=(0,0)$. On the other hand, if $\left(\hat{z}_{0}, \hat{v}_{0}\right)=(0,0)$, then

$$
\frac{1}{\tau} J\left(\left(\tau y_{d}, \tau u_{d}\right)\right) \geq 0, \quad \tau>0
$$

i.e.

$$
\frac{\tau}{2} \iint_{D_{T}}\left|\mathscr{L}\left(\left(y_{d}, u_{d}\right)\right)(x, t)\right|^{2} d x d t+\varepsilon\left\|\left(y_{d}, u_{d}\right)\right\|_{\mathscr{H}}-\left\|\left(y_{d}, u_{d}\right)\right\|_{\mathscr{H}}^{2} \geq 0, \quad \tau>0
$$

Letting $\tau \rightarrow 0^{+}$yields $\left\|\left(y_{d}, u_{d}\right)\right\|_{\mathscr{H}} \leq \varepsilon$.

Now, we are ready to prove the approximate controllability of the system (1.1)-(1.7).

Theorem 2.2 Assume $a_{j} \in C\left(\bar{Q}_{T}\right)$ is positive in $\Omega \times(0, T)$ with $\frac{1}{a_{j}} \frac{\partial a_{j}}{\partial t} \in L^{\infty}\left(Q_{T}\right)$ and $c_{j} \in L^{\infty}\left(Q_{T}\right)$ for $j=1,2$. The system (1.1)-(1.7) is approximately controllable. That is to say, for any given $y_{0}, u_{0}, y_{d}, u_{d} \in L^{2}(\Omega)$ and $\varepsilon>0$, there exists $h \in L^{2}\left(Q_{T}\right)$ such that the weak solution $(y, u)$ to the problem (1.1)-(1.6) satisfies (1.7).

Proof Since equations (1.1), (1.2) are linear and the terminal data $y_{d}, u_{d}$ are arbitrary, one can assume that

$$
y_{0}(x)=0, \quad u_{0}(x)=0 \quad \text { a.e. } x \in \Omega
$$


without loss of generality. Otherwise, one can divide $(y, u)$ into two solutions; one solves the fixed system with nonhomogeneous initial data and the other solves the control system with homogeneous initial data.

Let $\left(\hat{z}_{0}, \hat{v}_{0}\right)$ be the unique point of minimum of $J(\cdot)$ and denote by $(\hat{z}, \hat{v})$ the weak solution of the conjugate problem (2.1)-(2.6) with $\left(z_{0}, v_{0}\right)=\left(\hat{z}_{0}, \hat{v}_{0}\right)$. Below, let us show that $h=\hat{z}$ is a control to the system (1.1)-(1.7) under the assumption (2.10) by distinguishing into two cases.

The case $\left\|\left(y_{d}, u_{d}\right)\right\|_{\mathscr{H}} \leq \varepsilon$. In this case, Proposition 2.2 yields $\left(\hat{z}_{0}, \hat{v}_{0}\right)=(0,0)$ a.e. in $\Omega$ and thus $(\hat{z}, \hat{v})=(0,0)$ a.e. in $Q_{T}$ from the uniqueness result in Theorem 2.1. Therefore $h=0$ a.e. in $Q_{T}$ and thus $(y, u)=(0,0)$ a.e. in $Q_{T}$, which leads to

$$
\left\|\left(y(\cdot, T)-y_{d}(\cdot), u(\cdot, T)-u_{d}(\cdot)\right)\right\|_{\mathscr{H}}=\left\|\left(y_{d}, u_{d}\right)\right\|_{\mathscr{H}} \leq \varepsilon .
$$

First of all, we have the case $\left\|\left(y_{d}, u_{d}\right)\right\|_{\mathscr{H}}>\varepsilon$. In this case, Proposition 2.2 yields $\left(\hat{z}_{0}, \hat{v}_{0}\right) \neq$ $(0,0)$. For any $\left(\theta_{0}, \psi_{0}\right) \in \mathscr{H}$, denote by $(\theta, \psi)$ the weak solutions of the conjugate problem (2.1)-(2.6) with $\left(z_{0}, v_{0}\right)=\left(\theta_{0}, \psi_{0}\right)$. Since $\left(\hat{z}_{0}, \hat{v}_{0}\right)$ is the unique point of minimum of $J(\cdot)$, one gets

$$
\int_{0}^{T} \int_{\omega_{1}} \hat{z}(x, t) \theta(x, t) d x d t+\varepsilon \frac{\left\langle\left(\hat{z}_{0}, \hat{v}_{0}\right),\left(\theta_{0}, \psi_{0}\right)\right\rangle_{\mathscr{H}}}{\left\|\left(\hat{z}_{0}, \hat{v}_{0}\right)\right\|_{\mathscr{H}}}-\left\langle\left(y_{d}, u_{d}\right),\left(\theta_{0}, \psi_{0}\right)\right\rangle_{\mathscr{H}}=0
$$

It follows from the definition of the weak solution $(y, u)$ to the problem (1.1)-(1.6) with (1.7) and $h=\hat{z}$ that

$$
\begin{aligned}
& \iint_{Q_{T}} \frac{\partial y}{\partial t} \theta d x d t+\iint_{Q_{T}} a_{1} \nabla y \cdot \nabla \theta d x d t+\iint_{Q_{T}} c_{1} y \theta d x d t \\
& \quad=\iint_{Q_{T}} \hat{z} \chi_{\omega_{1}} \theta d x d t, \\
& \iint_{Q_{T}} \frac{\partial u}{\partial t} \psi d x d t+\iint_{Q_{T}} a_{2} \nabla u \cdot \nabla \psi d x d t+\iint_{Q_{T}} c_{2} u \psi d x d t \\
& \quad=\iint_{Q_{T}} y \chi_{\omega_{2}} \psi d x d t .
\end{aligned}
$$

Additionally, the definition of the weak solution $(\theta, \psi)$ of the problem (2.1)-(2.6) gives

$$
\begin{aligned}
& \iint_{Q_{T}} \theta \frac{\partial y}{\partial t} d x d t+\iint_{Q_{T}} a_{1} \nabla \theta \cdot \nabla y d x d t+\iint_{Q_{T}} c_{1} \theta y d x d t \\
& \quad=\iint_{Q_{T}} \psi \chi_{\omega_{2}} y d x d t+\int_{\Omega} \theta_{0}(x) y(x, T) d x \\
& \iint_{Q_{T}} \psi \frac{\partial u}{\partial t} d x d t+\iint_{Q_{T}} a_{2} \nabla \psi \cdot \nabla u d x d t+\iint_{Q_{T}} c_{2} \psi u d x d t \\
& \quad=\int_{\Omega} \psi_{0}(x) u(x, T) d x .
\end{aligned}
$$

From (2.12)-(2.15), one can get

$$
\iint_{Q_{T}} \hat{z} \chi_{\omega_{1}} \theta d x d t=\int_{\Omega} \psi_{0}(x) u(x, T) d x+\int_{\Omega} \theta_{0}(x) y(x, T) d x .
$$




\section{Combining (2.16) with (2.11) yields}

$$
\int_{\Omega}\left(y_{d}(x)-y(x, T)\right) \theta_{0}(x) d x+\int_{\Omega}\left(u_{d}(x)-u(x, T)\right) \psi_{0}(x) d x=\varepsilon \frac{\left\langle\left(\hat{z}_{0}, \hat{v}_{0}\right),\left(\theta_{0}, \psi_{0}\right)\right\rangle_{\mathscr{H}}}{\left\|\left(\hat{z}_{0}, \hat{v}_{0}\right)\right\|_{\mathscr{H}}},
$$

which implies (1.7) due to the arbitrariness of $\left(\theta_{0}, \psi_{0}\right) \in \mathscr{H}$.

\section{Competing interests}

The authors declare that they have no competing interests.

\section{Authors' contributions}

All authors contributed equally to the writing of this paper. All authors read and approved the final manuscript.

\section{Author details}

'College of Science, Changchun University, Changchun, 130022, P.R. China. ${ }^{2}$ School of Basic Science, Changchun University of Technology, Changchun, 130012, P.R. China. Institute of Mathematics, Jilin University, Changchun, 130012, P.R. China.

\section{Acknowledgements}

The authors are grateful to the anonymous referees for useful comments and suggestions, which improved the exposition of the paper. This research is supported by the National Natural Science Foundation of China (11401049), the Scientific and Technological Research Project of Jilin Province's Education Department (no. 2016285), the Twelfth Five-Year Plan project of Jilin Province's Educational Science (ZD14078), and SRF, JPED [2014](B019).

Received: 25 August 2015 Accepted: 29 June 2016 Published online: 11 July 2016

\section{References}

1. Martinez, P, Raymond, JP, Vancostenoble, J: Regional null controllability of a linearized Crocco-type equation. SIAM J. Control Optim. 42, 709-728 (2003)

2. Yin, J, Wang, C: Evolutionary weighted $p$-Laplacian with boundary degeneracy. J. Differ. Equ. 237(2), 421-445 (2007)

3. Fujii, N, Sakawa, Y: Controllability for nonlinear differential equations in Banach space. Autom. Control Theory Appl. 2(2), 44-46 (1974)

4. Fabre, C, Puel, J-P, Zuazua, E: Approximate controllability of a semilinear heat equation. Proc. R. Soc. Edinb., Sect. A, Math. 125(1), 31-61 (1995)

5. Russell, DL: Controllability and stabilizability theorems for linear partial differential equations: recent progress and open questions. SIAM Rev. 20(4), 639-739 (1978)

6. Alabau-Boussouira, F, Cannarsa, P, Fragnelli, G: Carleman estimates for degenerate parabolic operators with applications to null controllability. J. Evol. Equ. 6(2), 161-204 (2006)

7. Cannarsa, P, Fragnelli, G, Rocchetti, D: Controllability results for a class of one-dimensional degenerate parabolic problems in nondivergence form. J. Evol. Equ. 8(2), 583-616 (2008)

8. Cannarsa, P, Fragnelli, G, Vancostenoble, J: Regional controllability of semilinear degenerate parabolic equations in bounded domains. J. Math. Anal. Appl. 320(2), 804-818 (2006)

9. Cannarsa, P, Martinez, P, Vancostenoble, J: Carleman estimates for a class of degenerate parabolic operators. SIAM J. Control Optim. 47(1), 1-19 (2008)

10. Cannarsa, P, Martinez, P, Vancostenoble, J: Null controllability of degenerate heat equations. Adv. Differ. Equ. 10(2), 153-190 (2005)

11. Cannarsa, P, Martinez, P, Vancostenoble, J: Persistent regional controllability for a class of degenerate parabolic equations. Commun. Pure Appl. Anal. 3(4), 607-635 (2004)

12. Cannarsa, P, de Teresa, L: Controllability of 1-D coupled degenerate parabolic equations. Electron. J. Differ. Equ. 2009, 73 (2009)

13. Du, R, Wang, C: Null controllability of a class of systems governed by coupled degenerate equations. Appl. Math. Lett. 26(1), 113-119 (2013)

14. Martinez, P, Vancostenoble, J: Carleman estimates for one-dimensional degenerate heat equations. J. Evol. Equ. 6(2), 325-362 (2006)

15. Wang, C: Approximate controllability of a class of degenerate systems. Appl. Math. Comput. 203(1), 447-456 (2008)

16. Wang, C: Approximate controllability of a class of semilinear systems with boundary degeneracy. J. Evol. Equ. 10(1), 163-193 (2010)

17. Saut, JC, Scheurer, B: Unique continuation for some evolution equations. J. Differ. Equ. 66(1), 118-139 (1987) 